\title{
Nanokeramik aus der Kugelmühle
}

\author{
Chemiker des Max-Planck-Instituts für Kohlenforschung in Mülheim an der Ruhr haben einen \\ Weg gefunden, Korund, eine besonders stabile Variante von Aluminiumoxid, in Form von \\ Nanoteilchen herzustellen, und zwar durch simple Mechanochemie in einer Kugelmühle. Die \\ Teilchen könnten unter anderem als widerstandsfähiges Trägermaterial in Autokatalysatoren \\ oder als Ausgangsmaterial für besonders harte Keramiken dienen. Ein erstes Industrieunter- \\ nehmen arbeitet schon daran, nach dem Mülheimer Rezept zu produzieren.
}

In seinen edelsten Varianten formt Korund dank Spuren von Chrom, Eisen oder Titan Rubine und Saphire. Materialwissenschaftler interessieren sich für ihn aber weniger als Schmuckstein. Da Korund es in puncto Härte beinahe mit Diamant aufnehmen kann und auch gegen Hitze und Chemikalien ausgesprochen beständig ist, wird er nicht nur für keramische Implantate in der Zahnheilkunde, sondern auch für Prothesen oder Schneidwerkzeuge verwendet. Noch bruchfester ließe sich die Keramik machen, wenn sie aus Nanopartikeln des Aluminiumoxids produziert würde. Ein solches Herstellungsverfahren bräuchte zudem weniger Energie. Vereinfachen könnten Nanopartikel aus Korund auch den Bau von Autokatalysatoren, deren katalytisch aktive Kompo-

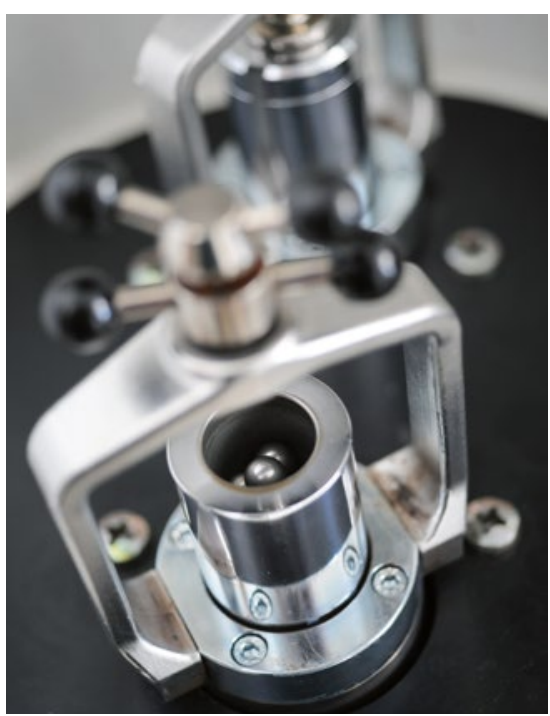

Bild 1 Aus Böhmit, einem wasserhaltigen Aluminiumoxid, entstehen Korund-Nanopartikel mit einer Oberfläche von $140 \mathrm{~m}^{2} \mathrm{~g}^{-1}$, wenn er etwa drei Stunden lang gemahlen wird. (๑ Frank Vinken/ MPI für Kohlenforschung) nente damit zudem stabiler würde. Bislang setzt die Automobilindustrie dafür in einem aufwendigen Verfahren eine weniger stabile Form von Aluminiumoxid ein.

Auch für die Chemieindustrie ist das besonders harte Aluminiumoxid in Form winziger Teilchen sehr interessant. „Es gibt Berichte, dass Katalysatoren mit einem Trägermaterial aus Korund-Nanopartikeln für die Produktion von Ammoniak effizienter arbeiten", sagt Ferdi Schüth, Direktor am Max-Planck-Institut für Kohlenforschung. „Bei anderen katalytischen Prozessen, wie etwa bei der Herstellung von synthetischen Kraftstoffen, könnte die höhere Stabilität wesentlich sein." Nanokristalle aus Korund finden künftig also möglicherweise zahlreiche Anwendungen - jetzt da die Mülheimer Chemiker einen Weg gefunden haben, sie durch Mechanochemie herzustellen.

Ein Pulver der winzigen Korundpartikel erhalten die Forscher, indem sie Brocken von Böhmit, einem wasserhaltigen Aluminiumoxid, das in dem häufig vorkommenden Erz Bauxit enthalten ist, schlicht drei Stunden lang in einer Kugelmühle mahlen und anschließend kurz erhitzen (Bild 1). Bislang konnten Chemiker Korund aus anderen Oxiden von Aluminium nur erzeugen, wenn sie die Ausgangsstoffe bei mehr als 1000 Grad Celsius brannten oder bei eher milden Temperaturen von um die $500^{\circ} \mathrm{C}$ wochenlang unter hohen Druck setzten. Dann bildeten sich zudem keine Nanokristalle, sondern größere Partikel. „Dass Nanopartikel aus Korund in einer Kugelmühle entstehen, haben wir zufällig festgestellt", sagt Ferdi Schüth. Sein Team untersuchte nämlich, ob eine katalytische Reaktion in einer solchen Mühle besser abläuft, weil der Katalysator beim Mahlen immer wieder eine frische Oberfläche erhält, an der die Reaktionspartner zueinanderkommen können. Als Katalysator verwendeten sie dabei ein weiches mit Goldpartikeln versetztes Aluminiumoxid und verfolgten das Geschehen in der Kugelmühle mit verschiedenen analytischen Methoden. Die offenbarten: Nach ein paar Stunden hatte sich ein Teil des Aluminiumoxids in Korund umgewandelt. „Das haben wir dann systematisch untersucht und dabei verschiedene Varianten des Aluminiumoxids als Ausgangsstoffe getestet", sagt Amol Amrute, einer der führenden Wissenschaftler in diesem Projekt.

Inzwischen können die Chemiker auch erklären, warum ein so banaler Vorgang wie das Mahlen eine Route zu einem Mineral eröffnet. Zum einen beeinträchtigen die Defekte wie Risse, Brüche und Stufen, die beim Mahlen entstehen, die Stabilität von Korund weniger als die von weicheren Aluminiumoxiden. Korund entsteht deshalb bevorzugt. Zum anderen liefern die Stöße, die das Material in der Mühle erfährt, genau die mechanische Energie, die für den ziemlich aufwendigen Umbau der Kristallstruktur zum Korund nötig ist. Die vergleichsweise niedrigen Temperaturen bei dem Prozess verhindern zudem, dass sich die Nanoteilchen zu größeren Körnern verklumpen. Nun untersuchen die Mülheimer Forscher, die auf die Entwicklung neuer Katalysatoren spezialisiert sind, wie sich Nanokorund als Katalysatormaterial in verschiedenen Reaktionen macht, zum Beispiel bei der Gewinnung synthetischer Kraftstoffe.

Kontakt:

Max-Planck-Institut für Kohlenforschung,

Mülheim an der Ruhr, www.kofo.mpg.de 